\title{
Cyclophosphamide Pharmacokinetics in Mice: A Comparison Between Retro Orbital Sampling Versus Serial Tail Vein Bleeding
}

\author{
Rana Said $^{\mathrm{a}, \mathrm{b}}$, Mohamed Abdel-Rehim ${ }^{\mathrm{c}, \mathrm{d}}$, Behnam Sadeghi ${ }^{\mathrm{a}, \mathrm{b}}$, Suleiman Al-Hashemi ${ }^{\mathrm{a}, \mathrm{b}}$, \\ Zuzana Hassan ${ }^{\mathrm{a}, \mathrm{b}}$ and Moustapha Hassan ${ }^{*}$,a,b \\ ${ }^{a}$ Department of Laboratory Medicine, Karolinska Institute, Stockholm, Sweden \\ ${ }^{b}$ Experimental Cancer Medicine, Clinical Research Center, Karolinska University Hospital-Huddinge, Sweden \\ ${ }^{c}$ Astrazeneca, $R \& D$, Södertälje, DMPK\&BAC, 15185 Södertälje, Sweden \\ ${ }^{d}$ Department of Chemistry, Karlstad University, 65188 Karlstad, Sweden
}

\begin{abstract}
Preclinical studies on pharmacokinetics in animals usually require at least 3-5 animals per time point. The use of several animals in each time point may increase the result variation due to individual dosing errors and inter- and intraindividual variation among animals. Moreover, a large number of animals has to be euthanized in these experiments.

In the present paper, the pharmacokinetics of the anticancer drug, cyclophosphamide was investigated using serial bleeding from the tail vein and compared with the kinetics obtained from traditional retro-orbital sinus bleeding in mice. Cyclophosphamide $(100 \mathrm{mg} / \mathrm{kg})$ was administered intraperitoneally to two groups of mice. Blood samples were collected at $0,0.25,0.5,1,2,3,4,5$ and $6 \mathrm{hr}$. In the first group $(\mathrm{n}=6), 20 \mu \mathrm{L}$ blood samples were collected from the tail-vein by serial bleeding. In the second group, 3 animals were killed at each time point and blood was collected from both tail vein and retro-orbital sinus. Cyclophosphamide analysis in whole blood was carried out using LC-MS/MS with on-line sample preparation utilizing microextraction by packed sorbent (MEPS). Pharmacokinetics including AUC, $\mathrm{t}_{\max }, \mathrm{C}_{\max }$ and half life were estimated using WinNonLin.

Cyclophosphamide concentrations in blood obtained after sampling through tail vein were close to those obtained after retro-orbital bleeding within the same animal. No significant differences in estimated pharmacokinetic parameters were found when serial tail vein bleeding was compared to retro-orbital bleeding.

Our results indicate that serial sampling using tail vein in mice can be a good alternative to retro-orbital sampling method. The method is reliable, easy and can be used for early preclinical kinetic studies. However, more studies are needed to evaluate different drug categories.
\end{abstract}

Keywords: Cyclophosphamide, pharmacokinetics, retro-orbital bleeding, tail-bleeding, LC/MS/MS.

\section{INTRODUCTION}

Pharmacokinetic, pharmacodynamic and recently pharmacogenetic studies in preclinical stage of drug development are important issues that have been discussed over the years. Investigation of the pharmacokinetic parameters requires blood sampling at several time points over the appropriate time period $[1,2]$. Thus, proper blood-collection procedures are critical for further drug development.

Mice and rats are frequently used in early pharmacokinetic studies. Mice have been one of the species of choice in toxicological evaluations due to low cost, minimal holding space, relatively short life span $[2,3]$, and lately because of the fact that mouse genome has been explored.

Unfortunately, there are no recommended standardized methods for blood sampling in animals that can be adapted by medical and/or pharmaceutical research $[4,5]$. Methods used today for blood sampling for pharmacokinetic studies vary depending on the animal species, frequency of blood

*Address correspondence to this author at the Experimental Cancer Medicine, Clinical Research Center, Novum, Karolinska University HospitalHuddinge, S-141 86 Stockholm, Sweden; Tel: +46-8-58583862; Fax: +468-58583810; E-mail: moustapha.hassan@ki.se collection, required blood volumes, researchers experience with blood collection and the nature of experiment [6].

Pharmacokinetic investigations require several samples at different time points and relatively large blood volumes for analysis [7]. Thus, in pharmacokinetic studies in mouse or rat a large number of animals are needed. However, using several animals at each time point may lead to high variation in the data and interpretation difficulties due to interindividual variation as expressed as differences in the expression of drug metabolism enzymes and genetic polymorphism [8-10].

Retro-orbital sinus bleeding technique is one of the most commonly used sampling methods for pharmacokinetic and toxicokinetic studies in mice. The method is invasive and requires appropriate anesthesia, monitoring and often veterinary assistance due to complications [11]. Moreover, it is becoming more difficult to obtain the ethical approval for retro-orbital bleeding. Nevertheless, the method can result in high volume of blood, but the number of samples taken from one animal is limited. As a result, several animals are needed for blood-collection to complete a pharmacokinetic concentration- time curve [12]. 
Different techniques have been employed to lesser extent to investigate the pharmaco- or toxokinetics such as microdialysis, cardiac puncture and mice -tail sectioning (tail clip). Some investigators have reported the use of the jugular and saphenous vein puncture methods. These techniques require shaving the ventral neck and upper thorax or the tarsal joint area that has to be carried out by well trained and experienced personal to assure successful serial blood sampling and minimize the infection risk for the animal [13].

Another used method is the tail-artery or -vein bleeding technique that does not require either anesthesia or preparation of the animal. In this method an incision at the end of a mouse or rat-tail is done followed by a gentle pressure along the tail from the tail bottom towards the tail top and the blood collected into a tube. The procedure is easy to perform and allows serial collection of several samples at different time points from the same animal $[11,14,15]$. However, this method is not widely used and is mostly applied in rats. This is due to the fact that the bioanalytical methods usually require large sample volumes. During the past years new applications of the liquid chromatography in combination with mass spectrometry (LC/MS) have stimulated development of more sensitive analysis of candidate drugs in small volumes ( $\mu \mathrm{L}$ scale) and at low concentrations (pg range) with high precision and accuracy $[15,16]$.

Several studies have been reported aiming to optimize blood sampling in a rapid and reproducible manner without affecting the kinetic profiles of the compound studied or disrupting physiological processes in the animal [15, 17-20]. However, there are only few studies on the effect of different blood sampling techniques on pharmacokinetic parameters of compounds in mice.

In the present study we compared the effect of two different blood sampling techniques on estimated pharmacokinetic parameters in MRI male mice. We have used anticancer drug cyclophosphamide that is a polar substance and has a low distribution volume. Serial tail vein bleeding using one mouse per concentration-time curve was compared to retroorbital bleeding using several animals per time point. We also assessed if nature of the samples, i.e. whole blood or plasma may affect the concentration-time curve of cyclophosphamide.

\section{MATERIALS AND METHODS}

\subsection{Materials}

Cyclophosphamide was purchased from Sigma Chemical Company (St. Louis, MO, USA). Lidocaine-d3 was used as internal standard and obtained from AstraZeneca (Södertälje, Sweden). Acetonitrile, methanol, formic acid and ammonium hydroxide were obtained from Merck (Darmstadt, Germany). All chemicals were of HPLC analytical grade.

\subsection{Animals}

All animal experiments were approved by the regional ethics committee for animal research in accordance with the Animal Protection Act, the Animal Protection Regulation and the Regulation of the Swedish National Board for Laboratory Animals.

MRI male mice were purchased from B\&K Universal (Sweden). All experiments were carried out on animals of
10-12 weeks old with weight $30-40 \mathrm{~g}$. Mice were allowed to acclimatize for their surrounding for one week prior to the start of experiments. The animals were kept in fully acclimatized room at constant temperature and humidity on a 12 hours light/dark cycle and were fed standard pellet and water ad libitum.

\subsection{Experimental Design}

Mice were divided in two groups. Both groups were administered cyclophosphamide intraperitoneally at a dose of $100 \mathrm{mg} / \mathrm{kg}$, but differed in blood sampling technique. Blood samples were collected at $0,0.25,0.5,1,2,3,4,5$ and 6 hours. Group A consisted of six mice and the blood samples were collected serially from the tail vein. Briefly, mice were placed in a restrainer and the tail was swabbed with alcohol. Because of the relatively long time intervals between sampling time points, the animals were not maintained in restrainer. With a sharp surgical blade, an incision over the tail vein located approximately $1-2 \mathrm{~cm}$ from the tip was made. The tail was slightly "milked" by applying even pressure from the base to the end and about $20 \mu \mathrm{L}$ of blood was collected using micro-syringe ( $100 \mu \mathrm{L}$ insulin syringe) containing $80 \mu \mathrm{L}(0.5 \mathrm{M}$ EDTA). A new incision was made for each time point starting from the tail end towards the tail base. This procedure followed to eliminate the effect of coagulation cascade on the sampling procedure. After bloodcollection procedure, direct pressure was applied on the incision site with a clean cotton swab for about $10 \mathrm{~s}$ to stop the bleeding and allow for the blood to clot. Subsequent samples were collected at time points mentioned above.

In group $\mathrm{B}$, three animals were used per each time point and blood samples were collected simultaneously from retroorbital sinus and tail vein at time points described above. For blood sampling, animals were anesthetized using isoflurane and blood was collected by the traditional retro-orbital bleeding in EDTA vacutainer tubes. Prior to retro-orbital sampling, blood samples were collected from the tail vein using the same technique as for serial bleeding $(20 \mu \mathrm{L}+80 \mu \mathrm{L}$ EDTA). Each blood sample collected through retro-orbital method was divided into two parts for the analysis of cyclophosphamide concentrations in whole blood and in plasma. One part was diluted 1:4 with EDTA $(0.5 \mathrm{M})$ for assay in whole blood, while the other part was centrifuged at $3000 \mathrm{~g}$ for $5 \mathrm{~min}$ at $4^{\circ} \mathrm{C}$, plasma was separated and stored at $-20^{\circ} \mathrm{C}$ until assay.

\subsection{Microextraction by Packed Sorbent (MEPS) for LC- MS/MS}

Blood and/or plasma was obtained from control animals and the standard calibration curve was prepared within the concentration range $0.5-150 \mu \mathrm{g} / \mathrm{mL}(0.5,0.75,5.0,7.5,10.0$, $25.0,50.0,100.0$, and $150.0 \mu \mathrm{g} / \mathrm{mL}$ ). Quality control (QC) samples concentrations were 15,80 and $120 \mu \mathrm{g} / \mathrm{mL}$. Cyclophosphamide was determined in mice blood using microextraction by packed sorbent as online sample preparation with liquid chromatography in combination with mass spectrometry (LC-MS/MS). Blood samples were diluted 1:25 by water and extracted using MEPS in two steps. The first step included the conditioning of the MEPS sorbent manually using $50 \mu \mathrm{L}$ methanol followed by $50 \mu \mathrm{L}$ of water. 
In the second step, the MEPS syringe was connected to the autosampler and the spiked plasma or blood sample (25 $\mu \mathrm{L}$ ) was withdrawn into the syringe by the autosampler. The sorbent was then washed once with $100 \mu \mathrm{L}$ of water/methanol 95:5 (v/v) to remove proteins and other interferences. The analytes were then eluted by $40 \mu \mathrm{L}$ methanol/water 95:5 (v/v) directly into the liquid chromatography (LC) injector. A gradient LC was used and the analysis was carried out on a Zorbax $(50 \times 2.1 \mathrm{~mm}, \mathrm{SB}-\mathrm{C} 8,3.5 \mu \mathrm{m})$ column was obtained from Agilent (CA, USA) with a flow rate of $150 \mu \mathrm{L} / \mathrm{min}$. Mobile phase A was $0.1 \%$ formic acid in water and acetonitril 90:10 (v/v) and mobile phase B contained $0.1 \%$ formic acid in water and acetonitril 20:80 (v/v). The gradient started from $1.0 \%$ of phase B up to $50 \%$ from 1 to $4 \mathrm{~min}$ and then from 4 to $6 \mathrm{~min}$ to $90 \%$ of phase B and at 6.5 min, phase B was set at $1.0 \%$ again. The flow rate was $150 \mu \mathrm{L} / \mathrm{min}$ and sample volume was $40 \mu \mathrm{L}$. The sorbent was cleaned using $4 \times 250 \mu \mathrm{L}$ elution solution followed by $4 \times 250$ $\mu \mathrm{L}$ of the washing solution between every extraction. The cleaning step was not only to decrease the memory effects, but also functioned as conditioning step before the next extraction. All experi-ments were conducted using a triple quadrupole mass spectrometric instrument Micromass Ultima (Waters Corpo-ration, Manchester, UK) equipped with a Z-electrospray interface operated in positive ion mode. The scan mode was multiple reaction monitoring (MRM) using precursor ion at $(\mathrm{M}+1) \mathrm{m} / \mathrm{z}(\mathrm{m} / \mathrm{z}$ : 261 and 237.9) and after collisional dissociation the product ions 140 and 86 were used for quantification of cyclophosphamide and the internal standard (lidocaine-d3).

\subsection{LC-MS/MS Condition}

The ESI mass spectrometer was operated in the positive ion mode with a capillary voltage at $3.1 \mathrm{kV}$, nitrogen was used as both cone gas and desolvation gas at flow rate of 400 and $20 \mathrm{~L} / \mathrm{h}$, respectively. The source and the desolvation temperature were set at 150 and $300 \mathrm{C}^{\circ}$, respectively. The cone voltage was optimized to $27 \mathrm{~V}$. The scan mode was multiple reaction monitoring (MRM) using precursor ion at $(\mathrm{M}+1) \mathrm{m} / \mathrm{z}(\mathrm{m} / \mathrm{z}: 261$ and 237.9$)$ and after collisional dissociation the product ions 140 and 154 were used for quantification of cyclophosphamide and its internal standard.

\subsection{The Pharmacokinetic Analysis}

Pharmacokinetic analysis of cyclophosphamide concentration-time data in blood or plasma and estimation of the pharmacokinetic parameters (AUC, distribution volume, terminal half life, the maximum concentration and clearance) were performed using Gauss-Newton (Levenberg-Hartly) criteria and were fitted to one compartment open model. The pharmacokinetic modeling was performed using WinNonlin, Version 5.2 (Pharsight, Mountain View, CA, USA). The estimated kinetic parameters were compared between the different sampling groups and analyzed statistically using Mann-Whitney test. A p-value of $<0.05$ was considered to be significant.

\section{RESULTS}

The analytical method using LC-MS/MS was sensitive and robust. The standard curve was linear within the range $0.5-150 \mu \mathrm{g} / \mathrm{mL}\left(\mathrm{r}^{2}>0.99 ; \mathrm{n}=3\right)$. The inter- and intra- day variation was 9 and $11 \%$, respectively. No difference between recovery from plasma or blood was observed.

After the intraperitoneal administration of cyclophosphamide, blood concentrations were fitted to one compartment open model in both groups. Blood concentration-time curves for cyclophosphamide obtained after serial bleeding from tail vein and after the conventional retro-orbital bleeding are presented in Fig. $(\mathbf{1 A}, \mathbf{B})$, resp.

Pharmacokinetic parameters (AUC, $\mathrm{t}_{1 / 2}, \mathrm{C}_{\max }, \mathrm{Cl}, \mathrm{Vss}$ ) are summarized in Table 1. The pharmacokinetic parameters are calculated based on whole blood analysis obtained after serial bleeding from the tail-vein in group A and the whole blood and plasma analysis after retro-orbital bleeding or tailvein sampling (group B) following intraperitoneal administration of cyclophosphamide. The AUC estimated from whole blood concentration-time curve after serial bleeding $(68.9 \mu \mathrm{g} . \mathrm{hr} / \mathrm{mL})$ was not significantly different from that obtained after retro-orbital bleeding $(61.3 \mu \mathrm{g} . \mathrm{hr} / \mathrm{mL})$. No significant differences in AUCs in group B were observed when whole blood obtained from retro-orbital sinus, whole blood from tail vein and plasma obtained from retro-orbital sinus were compared $(61.3,56.6$ and $68.5 \mu \mathrm{g} . \mathrm{hr} / \mathrm{mL}$, respectively).

The terminal elimination half-life values $\left(t_{1 / 2}\right)$ after intraperitoneal administration of cyclophosphamide and using different bleeding techniques were within the range 0.49 $0.52 \mathrm{hr}$. No significant differences were observed when different sampling techniques or analysis of whole blood and plasma were compared.

The maximum concentration $\left(\mathrm{C}_{\max }\right)$ was about 109 $\mu \mathrm{g} / \mathrm{mL}$ after serial bleeding showing tendency $(\mathrm{p}=0.09)$ to be higher than that seen after retro-orbital bleeding. However, the $\mathrm{C}_{\max }$ found from tail vein sampling in group $\mathrm{B}$ was about $79 \mu \mathrm{g} / \mathrm{mL}$. No differences were observed in Cmax using plasma or whole blood.

The distribution volumes of cyclophosphamide following intraperitoneal administration of cyclophosphamide were not statistically different comparing the results from whole blood obtained by two bleeding methods in group A and B (44 and $60 \mathrm{~mL}$, respectively). Neither the distribution volumes obtained from plasma samples using retro-orbital bleeding or whole blood in tail.vein in group B were significantly different (51 $\mathrm{mL}$ and $65 \mathrm{~mL}$, respectively).

The clearance values varied from $63 \mathrm{~mL} / \mathrm{hr}$ in group A to $80 \mathrm{~mL} / \mathrm{hr}$ in group B. The results are presented in Fig. (2) and no significant differences between clearances obtained after different bleeding techniques were observed.

\section{DISCUSSION}

The development of new chemical entities especially aiming the treatment of cancer has been reviewed world wide. In a recent publication by Tomaszewski [21], the American medical authorities outline an appropriate animal models for preclinical studies of pharmacology and toxicology including the maximum tolerated dose, dose limiting toxicity, safe starting dose and the reversibility of adverse effects was reviewed.

Blood collection methods have been thoroughly discussed. Blood collection techniques vary within and among 
(A)

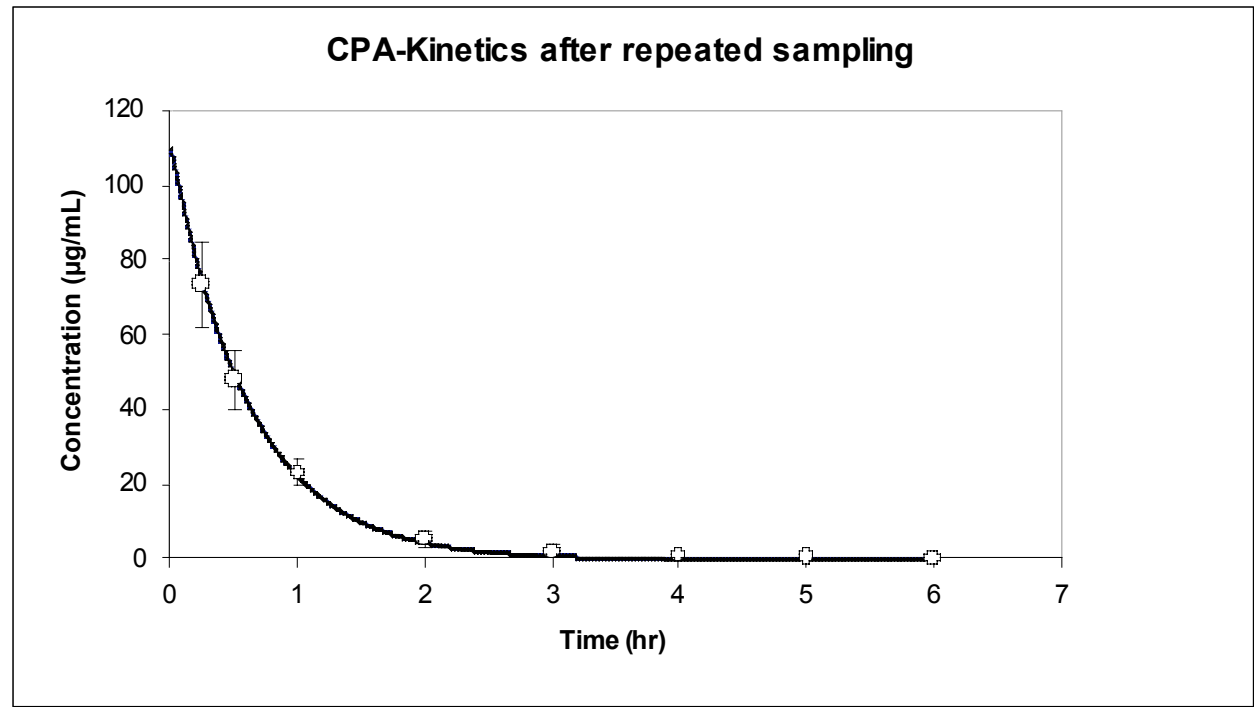

(B)

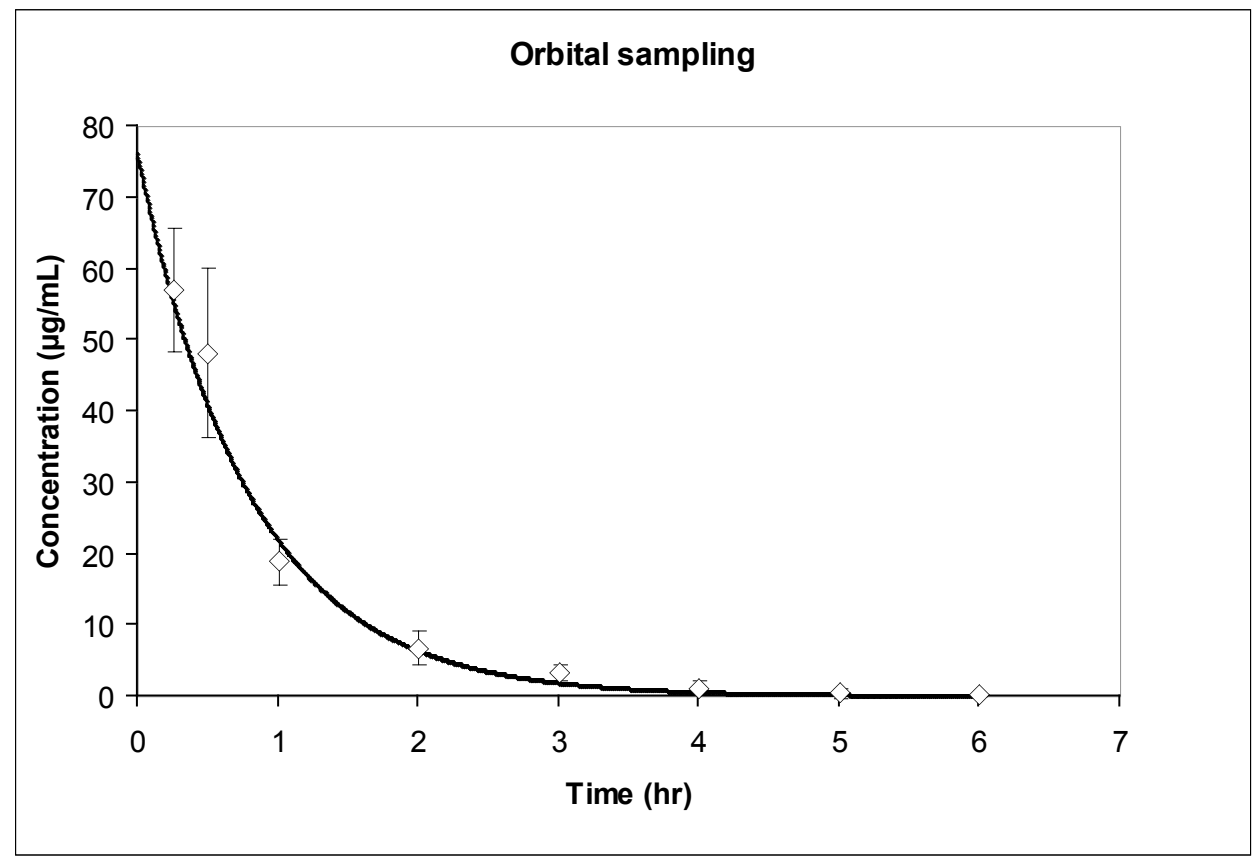

Fig. (1). Time versus concentration curve (A) obtained after the administration of cyclophosphamide (100mg/kg). (A) After serial bleeding from the tail vein in 6 mice, (B) obtained by orbital bleeding 3 mice in each time point. The solid line is the theoretical one compartment open model, $(\circ)$ in $1 \mathrm{~A}$ represent the mean of 6 mice for the experimental data \pm SD and $(\diamond)$ in 1 B represent the mean of 3 mice per time point for the experimental data $\pm \mathrm{SD}$.

species. Blood sampling using tail vein, jugular vein, saphenous vein and retro-orbital sinus are the most common bleeding sites. Mostly, these are invasive methods that can cause stress and may disturb the physiological status of the animal. Vachon et al. reported that repeated sampling from jugular vein in rat caused an increase in corticosterone levels [22] and Tabata et al. have reported an increase in blood glucose levels when blood samples were taken from mice tail by sectioning [23]. The validity of blood samples collected by the tail bleeding method has been questioned because of animal trauma, tail edema, and sample collection near the site of intravenous dosing. Therefore, retro-orbital sinus sampling technique is frequently chosen for blood collection in mice. However, this method does not allow the collection of several blood samples from the same animal. Moreover, it may cause several problems including damage for the optic nerve, ulceration and infection leading to ocular inflammation.

In the present study, we have compared serial tail bleeding using one mouse for the whole concentration-time curve with retro-orbital sampling using several mice per each time point. Moreover, we have compared the blood concentrations of a drug obtained using retro-orbital and tail vein sam- 
Table 1. Pharmacokinetic Parameters of Cyclophosphamide Calculated in Both Plasma and Blood After Different Samplings Procedures

\begin{tabular}{|c|c|c|c|c|}
\hline & Group A & \multicolumn{3}{|c|}{ Group B } \\
\hline & Serial Bleeding & \multicolumn{3}{|c|}{ Several Animals Per Time Point } \\
\hline & Tail Vein & Retro-Orbital Sinus & Tail Vein & Retro-Orbital Sinus \\
\hline & Whole Blood & Whole Blood & Whole Blood & Plasma \\
\hline PK parameters & Mean $\pm \mathrm{SD}$ & Mean \pm SD & Mean \pm SD & Mean \pm SD \\
\hline AUC ( $\mu \mathrm{g} / \mathrm{ml} . \mathrm{hr})$ & $68.9 \pm 10.5$ & $61.3 \pm 9.8$ & $56.6 \pm 13.8$ & $68.5 \pm 12.6$ \\
\hline $\mathrm{Cmax}(\mu \mathrm{g} / \mathrm{mL})$ & $108.8 \pm 18.2$ & $81.3 \pm 11.2$ & $78.7 \pm 24.6$ & $97.4 \pm 19.2$ \\
\hline $\mathrm{t}^{1} / 2(\mathrm{hr})$ & $0.45 \pm 0.08$ & $0.52 \pm 0.05$ & $0.51 \pm 0.08$ & $0.49 \pm 0.07$ \\
\hline $\mathrm{Cl}(\mathrm{mL} / \mathrm{hr})$ & $63 \pm 6$ & $80 \pm 9$ & $88 \pm 18$ & $72 \pm 10$ \\
\hline Vss (mL) & $44 \pm 9$ & $60 \pm 6$ & $65 \pm 15$ & $51 \pm 8$ \\
\hline
\end{tabular}

pling from the same animal. For this study, we have chosen cytostatic drug cyclophosphamide (CPA) because of its relatively long half-life, less effect of blood glucose or corticosterone on its metabolism and because of the low distribution volume of the drug. CPA is an alkylating agent and a prodrug undergoing a complicated process of metabolic activation and inactivation by cytochrome P450 enzymes, 4hydroxylation and $N$-dechloroethylation [24].

\section{CL}

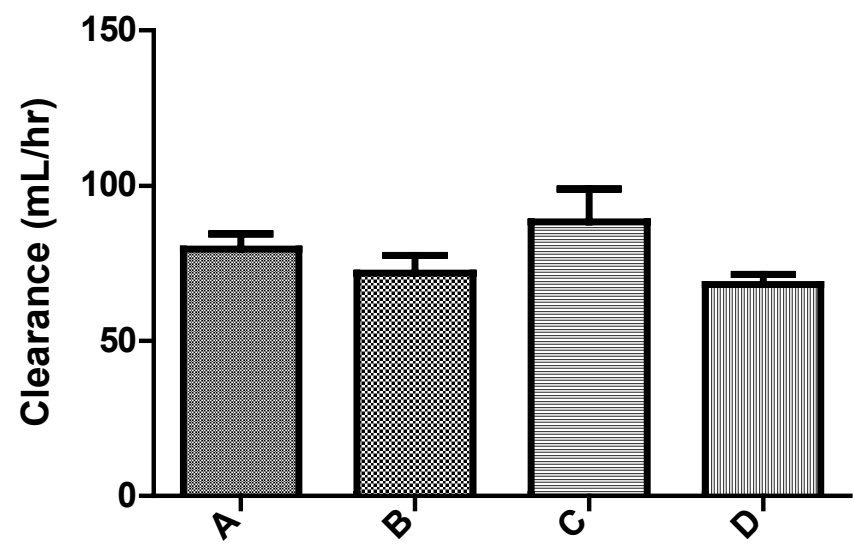

Fig. (2). Total body clearance $(\mathrm{mL} / \mathrm{hr})$ calculated after an IP injection of CPA $(100 \mathrm{mg} / \mathrm{kg})$ and followed by different sampling methods.

A: Clearance obtained in whole blood that was collected from retro orbital site (3 animals/each time point, group B).

B: Clearance obtained in plasma and blood was collected from retro orbital site (3 animals/each time point, group B).

$\mathrm{C}$ : Clearance obtained in whole blood that was collected from tail vein site (3 animals/each time point, group B).

D: Clearance obtained in whole blood that was collected from tail vein by serial bleeding ( 6 animals/each time concentration curve).

CPA is one of the most widely administered anticancer agents that is used in the treatment of hematological malignancies as well as solid tumors. CPA is also a potent immunosuppressive agent that affects both T-and B-lymphocytes, thus having effect on both humoral and cell-mediated immu- nity [25]. The doses of cyclophosphamide used in clinic are highly variable. As an immunosuppressive agent, CPA is administered orally in low dose $2-6 \mathrm{mg} / \mathrm{kg}$ body weight. In the treatment of neoplastic diseases a conventional intravenous doses of $500-1000 \mathrm{mg} / \mathrm{m}^{2}$ are usually used with 3-4 week intervals. CPA is also used in stem cell transplantation setting in high doses $\left(4-7 \mathrm{~g} / \mathrm{m}^{2}\right)[26]$.

In the present investigation we demonstrated that the pharmacokinetic parameters for cyclophosphamide obtained after serial tail-vein bleeding technique using full concentration-time curve from one animal are in a good agreement with those obtained after retro-orbital bleeding method using several animals per time-point. This is an important issue since CPA is one of the corner stones in cancer therapy and is activated through the CYP450 enzyme system and recently a number of pharmacogenetic studies have been reported. CYP450 is known to be polymorphic which require several animals at each time point to minimize the interindividual variation. Using serial bleeding technique in combination with an appropriate analytical method may allow researcher to achieve more reliable results with less variation.

A disadvantage of the tail-bleeding method may be that some drugs may affect the body temperature and hence the tail blood flow which in turn may affect the pharmacokinetics. It has also to be considered the number of incisions that has to be made on the tail. In our experiments we have chosen to make a new incision for each time point to eliminate the effect of the coagulation cascade on the pharmacokinetic parameters. However, no effect on the kinetics of cyclophosphamide was observed in other serial bleeding experiments where subsequent samples were obtained after reopening the first incision (data not shown). Another consideration in studies on metabolites with very short half-life is that the tail-vein bleeding can take longer time (up to $60 \mathrm{~s}$ ) compared to that using a catheter or retro-orbital sinus blood (20 to $40 \mathrm{~s})$.

In this paper we have shown that there is no significant difference in CPA blood concentrations obtained after retroorbital sampling compared with those obtained after sampling from tail vein from the same animal. Also estimated pharmacokinetic parameters were comparable. Despite that 
further studies are warranted to investigate different drugs with different physical and chemical properties. In a recent study, Hui et al. demonstrated that blood sampling methods in rats can affect the pharmacokinetic parameters depending on the drugs studied [18].

Serial tail-bleeding allows less blood volume samples per time point than does the major vein or retro-orbital bleeding method. However, due to recent technological improvements in sensitivity of bioanalytical methods such as normal phase and improved reverse phase LC-MS/MS much less plasma $(0.01-0.05 \mathrm{~mL})$ is needed to analyze the blood/plasma concentrations of compounds.

\section{SUMMARY}

Serial tail vein sampling requires lower number of animals for studies than other techniques requiring several animals in each time point. This allows for increased statistical power within a study and is particularly useful for toxicokinetic investigations. Moreover, serial tail-bleeding is less expensive as it requires only mouse restrainer during the blood collection. In contrast to the retro-orbital method, the tail-bleeding method allows the use of conscious mice and thus, use of general anesthesia may be avoided, and thereby the effect of anesthetic drug on metabolism of the studied drug. Finally, tail-bleeding allows for optimal pharmacokinetic and pharmacodynamic (PK/PD) study designs with parameters obtained within the same animal. In contrast, retro-orbital bleeding method is invasive, and thus, disruption of pharmacological end-points limits their utility in $\mathrm{PK} / \mathrm{PD}$ evaluations. Serial tail vein bleeding is easy to handle for researcher and with less stress for the animal, and allows for collection of as many as eight samples from a single mouse. One more important issue is that using the serial bleeding technique most likely will be considered to be more acceptable by the ethical authorities for animal welfare compared to retro-orbital bleeding.

\section{ACKNOWLEDGEMENTS}

The study was supported by grants from the Swedish Cancer Foundations and from the Swedish Children Cancer Society.

\section{REFERENCES}

[1] Baumgartner, G. Dtsch Tierarztl. Wochenschr., 1990, 97, 139-44.

[2] Rosner, F.; Bennett, A.J.; Cassell, E.J.; Farnsworth, P.B.; Halpern, A.L.; Landolt, A.B.; Loeb, L.; Numann, P.J.; Ona, F.V.; Risemberg, H.M.; Sechzer, P.H.; Sordillo, P.P.; Wolpaw, J.R. NY State J. Med., 1989, 89, 671-6.

[3] Cate, C.C. Lab. Anim. Care, 1969, 19, 256-8.

[4] Fluttert, M.; Dalm, S.; Oitzl, M.S. Lab. Anim., 2000, 34, 372-8.

[5] Chiou, W.L. Clin. Pharmacokinet., 1989, 17, 275-90.

[6] Malakoff, D. Science, 2000, 288, 248-53.

[7] Chiou, W.L. Clin. Pharmacokinet., 1989, 17, 175-99.

[8] Kerb, R.; Hoffmeyer, S.; Brinkmann, U. Pharmacogenomics, 2001, 2, 51-64.

[9] Darwish Marie, A.; Veggerby, C.; Robertson, D.H.; Gaskell, S.J.; Hubbard, S.J.; Martinsen, L.; Hurst, J.L.; Beynon, R.J. Protein Sci., 2001, 10, 411-7.

[10] Brinkmann, U.; Eichelbaum, M. Pharmacogenomics J., 2001, 1, 59-64.

[11] Khan, K.N.; Komoscar, W.J.; Das, I.; Lazzaro, N.C.; Senese, P.B.; Hamilton, P.; Roth, A.; Smith, P.F. Contemp. Top Lab. Anim. Sci., 1996, 35, 63-6.

[12] Durschlag, M.; Wurbel, H.; Stauffacher, M.; Von Holst, D. Physiol. Behav., 1996, 60, 1565-8.

[13] Hansen, D.K.; Davies, M.I.; Lunte, S.M.; Lunte, C.E. J. Pharm. Sci., 1999, 88, 14-27.

[14] Johannessen, W.M.; Tyssebotn, I.M.; Aarbakke, J. J. Pharm. Sci., 1982, 71, 1352-6.

[15] Xie, F.; Bruntlett, C.S.; Zhu, Y.; Kissinger, C.B.; Kissinger, P.T Anal. Sci., 2003, 19, 479-85.

[16] Bateman, K.P.; Castonguay, G.; Xu, L.; Rowland, S.; NicollGriffith, D.A.; Kelly, N.; Chan, C.C. J. Chromatogr. B. Biomed. Sci. Appl., 2001, 754, 245-51.

[17] de Pont, A.C.; Hofstra, J.J.; Pik, D.R.; Meijers, J.C.; Schultz, M.J. Crit. Care, 2007, 11, R102.

[18] Hui, Y.H.; Huang, N.H.; Ebbert, L.; Bina, H.; Chiang, A.; Maples, C.; Pritt, M.; Kern, T.; Patel, N. J. Pharmacol. Toxicol. Methods, 2007, 56, 256-64.

[19] Pass, G.J.; Carrie, D.; Boylan, M.; Lorimore, S.; Wright, E.; Houston, B.; Henderson, C.J.; Wolf, C.R. Cancer Res., 2005, 65, 42117.

[20] Watters, J.W.; Kloss, E.F.; Link, D.C.; Graubert, T.A.; McLeod, H.L. J. Appl. Physiol., 2003, 95, 1352-60.

[21] Tomaszewski, J.E. Eur. J. Cancer, 2004, 40, 907-13.

[22] Vachon, P.; Moreau, J.P. Contemp. Top. Lab Anim. Sci., 2001, 40, 22-4.

[23] Tabata, H.; Kitamura, T.; Nagamatsu, N. Lab. Anim., 1998, 32, 143-8.

[24] Sladek, N.E. Pharmacol. Ther., 1988, 37, 301-55.

[25] Binotto, G.; Trentin, L.; Semenzato, G. Oncology, 2003, 65 Suppl 2, 17-20.

[26] de Jonge, M.E.; Huitema, A.D.; van Dam, S.M.; Rodenhuis, S.; Beijnen, J.H. Anticancer Drugs, 2005, 16, 331-6. 